\title{
Social Media Sentimental Research using Machine Learning Techniques
}

\author{
G.Manoj Naidu, Bhuvaneswari Balachander
}

\begin{abstract}
Social media is the main resource to collect information about people opinion towards different topics as they spend most of their time on social media and share their thoughts. In this technical paper we present the applications of sentimental analysis. As we chosen twitter as our analysis platform we show how to connect to twitter and run analysis queries. We illustrate approach to issue with the model to different fields and show the best results.
\end{abstract}

Keywords: Social media, Sentimental analysis.

\section{INTRODUCTION}

Opinion mining is very important research area because due to the large amount of data is posted on social media. The people accessing to the internet are increasing day by day. It is difficult to provide best to the users without some analysis.

Sentimental analysis is a must required process in every filed of business to get feedback of their products by which organizations can take it and use in further development pf product.

The best approach to social media analysis allows the network through connections that develop with in. Twitter is used to track opinions in those cases in which changes to be done, So we focus more on twitter in this paper.

The text data of users is taken and provide the solution by summarizing and training it. The result is given whether the analysis is positive, negative or neutral. In this method words are tokenized from raw text data of users tweets.

Datatype comprehension is troublesome one to explain. Along these lines, AI procedures like managed and unsupervised calculations are being utilized. We are moving toward two kinds of investigation. First methodology is to construct word mists from clients data(tweets), and the second methodology is to show chart visuals of a specific point examining on informal organization through information. Few methods are been used to understand the text. We use natural language tool kit(NLTK) and logistic regression approach for sentimental analysis where it classifies text into binary classification( 0 or 1$)$. The text is classified into positive and negative one. Words with hate classifies as negative one. The graph visuals represent general report and detailed report where it shows people thoughts in percentage.

In this paper, we will examine online networking wistful examination and significance of it in different fields and how distinctively it tends to be drawn closer. And

Revised Manuscript Received on August 05, 2019.

G.Manoj Naidu, Saveetha institute of medical and technical sciences, Chennai, Tamil Nadu, India

Ms. Bhuvaneswari Balachander, Saveetha institute of medical and technical sciences, Chennai, Tamil Nadu, India furthermore about twitter customer key and access token which is rich asset for information and its examination.

\section{SOCIAL MEDIA ANALYSIS}

Internet based life wistful examination is the investigation of individuals assessment and thought on different subjects, patterns and these days it is must for business. Numerous individuals express their conclusions on numerous subjects on well known patterns via web-based networking media like twitter. Wistful examination has numerous advantages in business.

Twitter is the most prominent informal organization on hot patterns very day. Twitter is the rich asset to find out about individuals supposition and do nostalgic examination. All tweets on specific point can be determined as whether it is sure, negative, or unbiased.

Users generally use short cuts to spell a word. It becomes tough situation to mine the text. Natural language processing is used to solve this problem. It is done by using natural language tool kit.

\section{TWITTER SENTIMENTAL ANALYSIS}

Social media is the main resource to collect information about people opinion towards different topics as they spend most of their time on social media and share their thoughts. There are many opinion mining systems regarding different topics. This sentimental analysis will be beneficial in many businesses and politics.

Social media like twitter contains the large amount of data in the form of tweets. Usually people use many short words and abbreviations which is very difficult to extract text. Along with natural language processing we approach through machine learning techniques to make the operation easy and simple.

\section{IV. .DESIGN AND IMPLEMENTATION \& RESULTS}

This paper demonstrates the execution of twitter wistful examination by buyer key and access token which is given by twitter. There are many tools and best libraries are used in this project.

Implementation is mentioned in step by step method.

a. Downloading twitter dataset for classification.

b. Importing required libraries.

c. Cleaning dataset by replacing missing values with mean value and droping some columns which are not used. 
d. Tokenize the words from tweets and pass it to array.

e. Fit the whole data into machine learning model.

f. Showing the results in word clouds and graphs.

This method can be applied in any field for better results. For example we can use it in case of new phone which is released and new movie.

Cleaning the data takes the major time as tweets have handles like@abc. And analysis based upon positive, negative and neutral is done on hashtags.

Figure 1 shows the tidy tweets which is derived from original data after cleaning it.

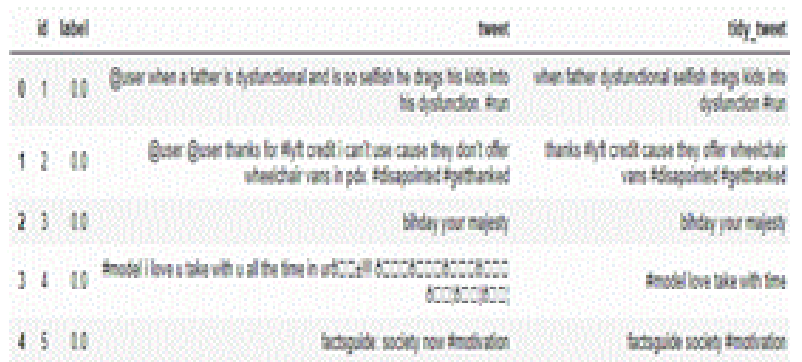

Figure 1 Cleaned data from raw data

\section{A. Implementation:}

In this project, we used python programming language for sentimental analysis. We used the following libraries:

1.Re

2.Pandas

3.Numpy

4.Matplotlib

5.Seaborn

6.Nltk

8.Tweepy

Anaconda navigator is best source for implementation of machine learning projects.

The textblob is python programming library for content preparing and it likewise uses note for characteristic language handling.

\section{B. Connect to twitter API:}

To connect to twitter live tweets and trends, we need to create account on twitter and define app. Users must go to apps.twitter.com/app/new and access to API keys.

\section{Sample results:}

Following results shows the sample output for particular topic called 'IAF' based on 100 tweets.

Enter Keyword/Tag to search about: IAF

Enter how many tweets to search: 100

How people are reacting on IAF by analyzing 100 tweets.

General Report:

Weakly Negative

Detailed Report:

$3.00 \%$ people thought it was positive

$11.00 \%$ people thought it was weakly positive

$2.00 \%$ people thought it was strongly positive

$10.00 \%$ people thought it was negative

$40.00 \%$ people thought it was weakly negative

$2.00 \%$ people thought it was strongly negative

$32.00 \%$ people thought it was neutral
7.Textblob

The actual positive tweets and negative tweets will be streamed and saved in file as csv or text format. I cannot mention it here as it is large amount of data.

Figure 2 demonstrates the example aftereffect of word cloud which depends on various sort of tweets from clients. Tokenized words sent to cluster and determined it as positive and negative names. Strategic relapse groups it into two sort of datasets in which one sort contains words with positive suppositions like love, great, extraordinary, marvelous and so forth. The second kind contains words with negative sentiments like cruel, awful, terrible and so on.

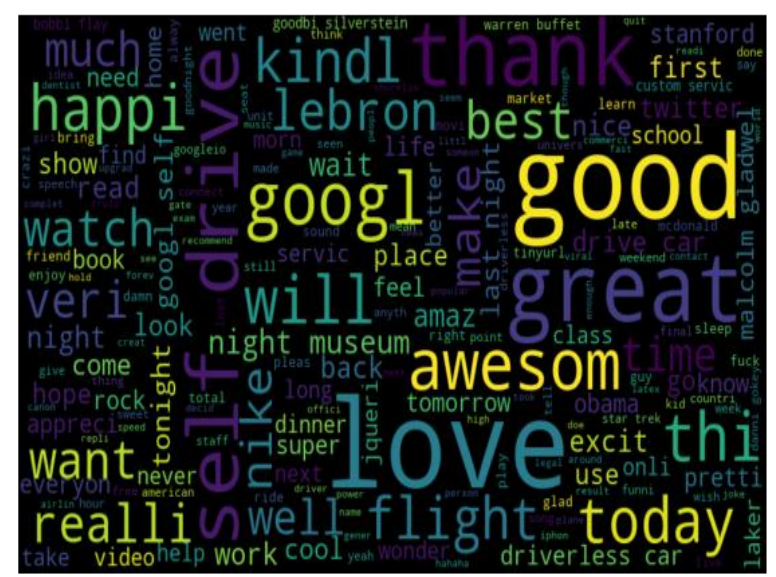

Figure 2 Word cloud

Figure 3 demonstrates the pie diagram which gets suppositions from tweets in three different ways that are sure, negative and nonpartisan. On the off chance that we run program in various occasions we may distinctive sort of results dependent on tweets at a specific time and conditions.

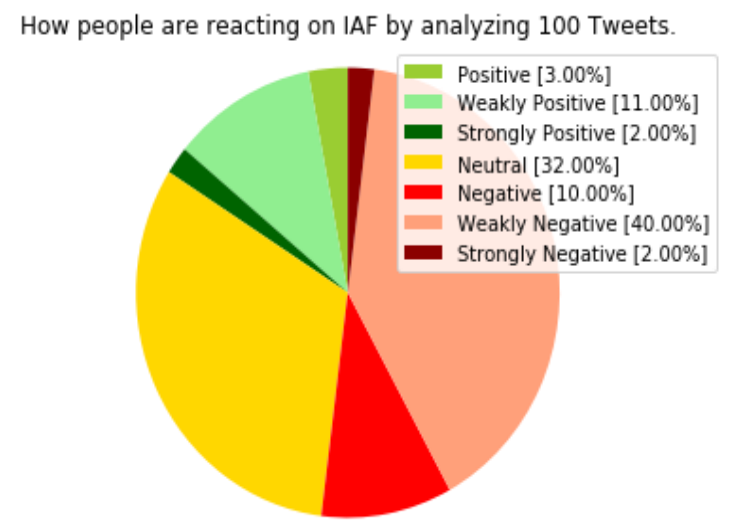

Figure 3 Sentiment analysis results.

\section{CONCLUSION}

In this technical paper, we discussed the importance of social media analysis and its applications in different fields. We used twitter and have implemented sentimental analysis using python programming language and showed the best results. It is also known that more implementation shows still better results. 


\section{REFERENCES}

1. Hamid Bhageri, Sentiment analysis of twitter data. (2017)

2. Paolo Fornacciari, Social Network and Sentiment Analysis on Twitter.

3. Varsha Sahayak, Sentimental analysis on twitter data.(2015)

4. ApoorvAgarwal, Sentimental analysis of twitter data.

5. Akshi Kumar and Teeja Mary Sebastia, Sentiment analysis on twitter.

6. Anusha K S1, Radhika A D, A Survey on Analysis of Twitter Opinion Mining Using Sentiment.(2017).

7. K.Nirmala Devi, Social Media Aided Sentiment Analysis in Forecasting.(2017).

8. Onam Bharti, SENTIMENT ANALYSIS ON TWITTER DATA.(2016).

9. Bharat Naiknaware1, Bindesh Kushwaha2, Seema Kawathekar, Social Media Sentiment Analysis using Machine Learning Classifiers.(2017).

10. Liu, B. (2012). Sentiment analysis and opinion mining Synthesis lectures on human language technologies, 5(1), $1-167$.

11. Agarwal, A, Xie, Vovsha, Rambow, O., \& Passonneau, R. 2011, June. Sentiment analysis of twitter data. In Proceedings of workshops on the languages in social media. Association with Computational Linguistics. 